Provided by the author(s) and University of Galway in accordance with publisher policies. Please cite the published version when available.

\begin{tabular}{|c|c|}
\hline Title & Promoting quality of life for patients with myeloma \\
\hline Author(s) & Dowling, Maura; Kelly, Mary; Meenaghan, Teresa \\
\hline $\begin{array}{c}\text { Publication } \\
\text { Date }\end{array}$ & 2013-08-02 \\
\hline $\begin{array}{l}\text { Publication } \\
\text { Information }\end{array}$ & $\begin{array}{l}\text { Dowling, Maura, Kelly, Mary, \& Meenaghan, Teresa. (2013). } \\
\text { Promoting quality of life for patients with myeloma. Cancer } \\
\text { Nursing Practice, 12(8), 30-37. doi: } \\
\text { 10.7748/cnp2013.10.12.8.30.e994 }\end{array}$ \\
\hline Publisher & RCN Publishing \\
\hline $\begin{array}{l}\text { Link to } \\
\text { publisher's } \\
\text { version }\end{array}$ & https://dx.doi.org/10.7748/cnp2013.10.12.8.30.e994 \\
\hline Item record & http://hdl.handle.net/10379/14804 \\
\hline DOI & http://dx.doi.org/10.7748/cnp2013.10.12.8.30.e994 \\
\hline
\end{tabular}

Downloaded 2023-04-26T10:17:09Z

Some rights reserved. For more information, please see the item record link above.

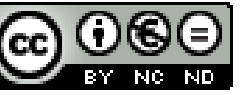




\section{Promoting quality of life for patients with myeloma}

Dowling, Maura, Kelly, Mary, \& Meenaghan, Teresa. (2013). Promoting quality of life for patients with myeloma. Cancer Nursing Practice, 12(8), 30-37. doi: 10.7748/cnp2013.10.12.8.30.e994

\section{Abstract}

This article aims to provide nurses with an update of current best practice in caring for patients living with a diagnosis of myeloma. New targeted treatments for myeloma have resulted in extended survival for patients. However, with this extended survival are both disease and treatment related side effects that have a profound effect on patients' quality of life. Nurses play a key role in supporting patients to manage their illness and treatment, and promote an improved quality of life.

Key words: bone health, myeloma, myelosuppression, peripheral neuropathy, renal failure. 


\section{Aims and intended learning outcomes}

The aim of this article is to provide nurses with an update of current best practice in caring for patients living with a diagnosis of myeloma. In recent years, new targeted treatments for myeloma have resulted in extended survival for patients; however, these treatments can have a profound effect on myeloma patients' quality of life. Nurses play a key role in helping myeloma patients understand and manage the side effects of their treatment, and ultimately improve patients' quality of life.

After reading this article and completing the time out activities, you should be able to:

- List the major side effects of treatments used in the management of myeloma;

- Describe the management of myelosuppression in myeloma;

- Summarise the management of peripheral neuropathy in myeloma;

- Outline the management of pain in myeloma;

- Discuss the management of bone health in myeloma;

- Summarise the management of renal complications in myeloma.

\section{Introduction}

Myeloma is a malignancy of the blood characterised by uncontrolled plasma cell proliferation (Palumbo et al 2011). The illness is characterised by a variety of symptoms, including bone pain, fatigue, hypercalcaemia, renal insufficiency and 
reduced blood counts leading to anaemia, increasing infection risk and increased possibility of bleeding (Coleman et al 2011).

Presenting signs and symptoms are generally vague and non-specific, which poses a challenge for early diagnosis and intervention. It is estimated that approximately $30 \%$ of new myeloma cases are diagnosed when patients present to the doctor with unrelated symptoms, such as fatigue and bone pain (George and Sadovsky, 1999).

Fatigue, bone pain, recurrent infections and alteration in kidney function can lead to increased hospital visits for transfusions, antibiotics and fluids. This often have a severe impact on patients' quality of life. Quality of life is therefore at the forefront of management of myeloma. Nursing management of myeloma is challenging, requiring ongoing assessment, evaluation and review. Specialist nurses in particular, play a key role in the provision of on-going care and support to myeloma patients on their treatment journey.

Although myeloma remains incurable, advances in treatments over the past two decades has resulted in improved prognosis for patients, with about $50 \%$ of patients achieving a complete response (Engelhardt et al 2010). However, because patients may require continuous long-term treatments to control their disease, a range of adverse events (AEs) can develop. Attention to these adverse events is essential, with early intervention to promote patients' QOL. In addition, because the median patient age at diagnosis is around 70 years with $37 \%$ of patients aged 75 years or over, the 
existence of co-morbidities adds to the risk of these adverse effects (Palumbo et al 2011).

\section{Time out 1}

Pause for a moment and make a list of the tests that you have used locally to diagnose myeloma. Try to summarise how the age of the patient and any existing morbidities affected the diagnostic work undertaken.

\section{Diagnosing myeloma}

Myeloma can be difficult to diagnose because the symptoms are often vague. If myeloma is suspected many investigations need to be carried out including a bone marrow biopsy, skeletal survey, many different types of blood tests (serum protein electrophoresis (SPEP), beta 2 microglobulin) serum free light chains (SFLC), and kidney function tests. See Box 1 for a list of investigations required for diagnosis.

When the bone marrow aspirate is taken cytogenetics can be requested. Cytogenetics (examining cell chromosomes), play a key role in the diagnosis, prognosis and treatment of myeloma (Jacobson et al 2012). In myeloma, the presence or absence of certain chromosomal abnormalities helps classify a patient's risk status and knowing a patient's risk status helps reach a decision regarding a treatment plan (Jacobsen et al 2012). High-risk patients are those with advancedstage disease (stage III according to the International Staging System); those with poor cytogenetics, such as $\mathrm{t}(4: 14), \mathrm{t}(14: 16)$, and $\mathrm{t}(14: 20)$, deletion of chromosome 13 , inactivation of TP53 (a tumour suppressor protein); and those with a complex 
karyotype. Patients with very high proliferative rates are also included in this classification.

\section{Box 1 Investigations indicated to diagnose myeloma}

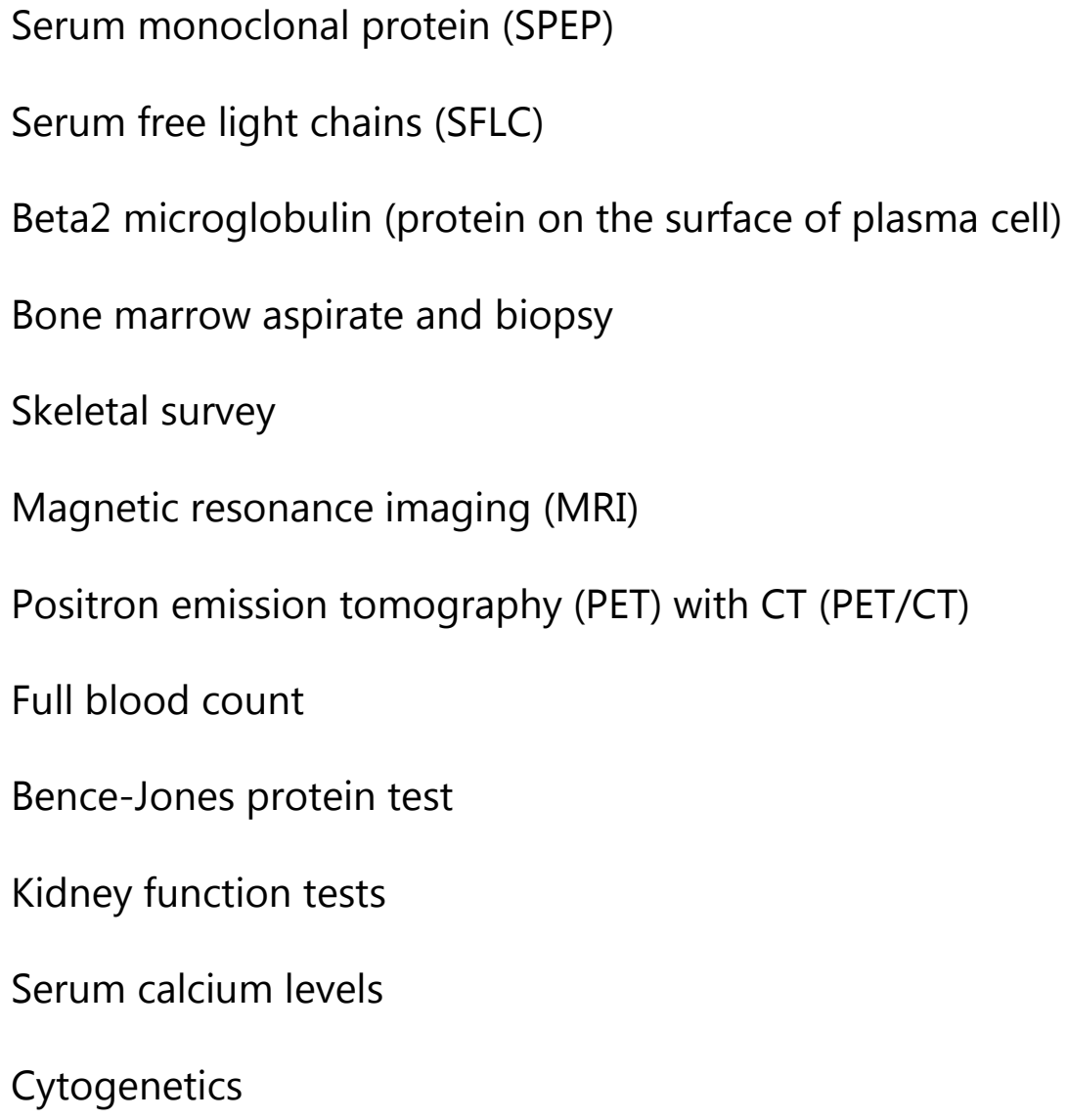

\section{Time out 2}

Return now to any patients that you remember and summarise the treatment options that were discussed with them. How were the options considered given the patient's existing health status and pre existing co morbidities?

\section{Current treatment options for myeloma patients}

There are a variety of treatment options used in the management of myeloma. The treatment choice is individualised to the patient's disease, performance status (Box 2) 
and transplant eligibility. Treatment options are also guided by Department of Health and NICE guidance (or local equivalent), drug licensing, financial issues and the patient's choice.

Box 2 ECOG Performance Status (Oken et al 1982)

\section{ECOG PERFORMANCE STATUS*}

\begin{tabular}{|l|l|}
\hline Grade & ECOG \\
\hline 0 & Fully active, able to carry on all pre-disease performance without restriction \\
\hline 1 & $\begin{array}{l}\text { Restricted in physically strenuous activity but ambulatory and able to carry } \\
\text { out work of a light or sedentary nature, e.g., light house work, office work }\end{array}$ \\
\hline 2 & $\begin{array}{l}\text { Ambulatory and capable of all selfcare but unable to carry out any work } \\
\text { activities. Up and about more than 50\% of waking hours }\end{array}$ \\
\hline 3 & $\begin{array}{l}\text { Capable of only limited selfcare, confined to bed or chair more than } 50 \% \text { of } \\
\text { waking hours }\end{array}$ \\
\hline 4 & $\begin{array}{l}\text { Completely disabled. Cannot carry on any selfcare. Totally confined to bed or } \\
\text { chair }\end{array}$ \\
\hline 5 & Dead \\
\hline
\end{tabular}

The introduction of immunomodulatory drugs (IMIDs), thalidomide, lenalidomide and pomalidomide and proteasome inhibitors, bortezomib and carfilzomib have offered extended survival for patients being treated for myeloma (Harousseau 2012). Evidence supports the use of drug combinations and all include steroid therapy. Many treatment regimens are used; a sample of these are outlined in table 1 . Patients with poor cytogenetics or who have had multiple relapses should continue on a treatment regimen as maintenance post transplant (Chang 2012). In addition, 
depending on a patient's overall response, consolidation treatment before starting maintenance may be required.

Numerous studies report that induction with thalidomide or bortezomib has consistently shown better complete responses $(C R)$ or very good partial response (VGPR) when compared against VAD (vincristine, doxorubicin, dexamethasone) based regimes (Harousseau 2012). Moreover, evidence also shows that triple combinations of thalidomide-bortezomib-dexamethasone results in the best $C R$ rate either before or after autologous stem-cell transplantation (ASCT) when compared to double combinations of either thalidomide-dexamethasone (TD) or bortezomibdexamethasone (VD) (Harousseau 2012).

Lenalidomide is effective in both newly diagnosed myeloma and relapsed/refractory myeloma (Quach et al 2012). In addition, lenalidomide is better tolerated than thalidomide and is recommended for maintenance treatment (Harousseau 2012). It is given as a single agent maintenance therapy or with low dose dexamethasone. However, all patients on lenalidomide or thalidomide with dexamethasone also require antithrombosis prophylaxis (Rajkumar 2013).

Haematopoietic stem cell transplantation is also a treatment option for myeloma patients. Autologous stem cell transplantation (ASCT) involves transplantation of the patient's own stem cells that have been previously harvested. ASCT does not provide a cure but it provides prolonged event-free survival. Eligibility for autologous stemcell transplantation (ASCT) is an important early decision following diagnosis 
(Harousseau 2012). Allogenic transplantation (from a healthy donor to the patient) remains a limited option and is not routinely considered, because it has been associated with excessively high rates of mortality (Shimoni et al 2010). The decision to undergo ASTC is based on a patient's age, performance status, co-morbidities and patient preference (Muta et al 2013). The timing of cell harvesting is also important because patients who receive multiple different treatments have poor harvesting; early harvesting after a few cycles of treatment is therefore advised. Patients are typically reassessed after four cycles of induction therapy before undergoing cell harvesting. After this point, patients can either have a transplant or delay transplant until first relapse and continue with induction therapy (Rajkumar 2013). Notably, a very good partial response (VGPR) after induction therapy is an important prognostic factor in patients undergoing ASCT (Moreau et al 2011a).

How long patients should remain on treatment is uncertain, and guidelines from a variety of sources (e.g. NICE guidance, drug manufacturers) are considered when reaching a decision. However, decisions also need to be individualised to each patient's needs, such as response and tolerance to treatments (Palumbo 2012). Specialist haematology nurses play an important education role in the early stages of treatment, clarifying treatment issues raised by the patient's consultant physician and educating the patient on expected symptoms. General nurses also play an important role at this time, re-emphasising self-care measures to minimise the side effects of treatment and assessing for these side effects. 
Table 1. Major treatment regimens in myeloma (adapted from Rajkmar 2013).

\begin{tabular}{|l|l|}
\hline Regimen & Comments \\
\hline Melphalan-prednisone & $\begin{array}{l}\text { Rarely used today without IMID except } \\
\text { in frail elderly or palliative setting. } \\
\text { MP induces a response in 50-60\% of } \\
\text { patients. Disappearance of the M } \\
\text { component on electrophoresis occurs } \\
\text { in only 3\% of patients, and cure is } \\
\text { extraordinarily rare (Seiter 2013). }\end{array}$ \\
\hline Thalidomide-dexamethasone (TD) & $\begin{array}{l}\text { Not recommended as front-line } \\
\text { treatment except in countries where } \\
\text { lenalidomide is not available for initial } \\
\text { treatment and in patients with acute } \\
\text { renal failure. } \\
\text { Anti-thrombosis prophylaxis needed } \\
\text { with thalidomide. }\end{array}$ \\
\hline Lenalidomide-dexamethasone & $\begin{array}{l}\text { Lenalidomide adversely affects stem } \\
\text { cell mobilisation. Therefore, it is } \\
\text { recommended that stem cells should } \\
\text { be collected within } 6 \text { months of } \\
\text { initiation of lenalidomide therapy } \\
\text { (Kumar et al 2007). } \\
\text { Bortezomib-dexamethasone (VD) } \\
\text { (VTD) }\end{array}$ \\
\hline Melphalan-prednisone-thalidomide lenalidomide. \\
\hline wortezomib-melphalan-prednisone & $\begin{array}{l}\text { Bortezomib does not adversely affect } \\
\text { stem cell mobilisation. }\end{array}$ \\
\hline & $\begin{array}{l}\text { Bortezomib acts a thromboprotective } \\
\text { agent and when used with }\end{array}$ \\
\hline
\end{tabular}




\begin{tabular}{|l|l|}
\hline & $\begin{array}{l}\text { thalidomide, reduces the risk of } \\
\text { venous thromboembolism. }\end{array}$ \\
\hline $\begin{array}{l}\text { Bortezomib-cyclophosphamide- } \\
\text { dexamethasone (VCD) }\end{array}$ & $\begin{array}{l}\text { Bortezomib-lenalidomide-dexamethasone } \\
\text { (VRD) }\end{array}$ \\
\hline $\begin{array}{l}\text { Bortezomib-dexamethasone-thalidomide- } \\
\text { cisplatin-doxorubicin-cyclophosphamide- } \\
\text { etoposide (VDT-PACE) }\end{array}$ & $\begin{array}{l}\text { Used in patients with aggressive } \\
\text { disease. }\end{array}$ \\
\hline
\end{tabular}

\section{Time out 3}

Look back at the myeloma treatments just outlined and identify what adverse effects patients can develop from these treatments. Thinking about patients that you have cared for, were any of the adverse effects especially challenging to manage? Try to summarise why.

\section{Effects associated with myeloma and its treatment}

Adverse effects (AEs) related to myeloma treatment are many. Common treatment related effects include myelosuppression and peripheral neuropathy (Mateos 2012).

\section{Myelosuppression}

Chemotherapy can affect the bone marrow resulting in a reduction in the number of blood cells. Symptoms related to the disease or its treatments often correlate with the blood counts that are affected the most. A reduction in red cells will often lead to symptoms such as fatigue or shortness of breath. These symptoms can be improved by transfusions or using erythropoietin (NICE 2008). However, it is important to 
monitor patients to ensure a safe $\mathrm{Hb}$ level is achieved because there is an increased risk of thrombotic events if a patient's $\mathrm{Hb}$ rises higher than $12 \mathrm{~g} / \mathrm{dl}$.

It is essential that both ward and specialist nurses encourage patients to talk about any symptoms of fatigue because patients are sometimes reluctant to do so with physicians in case their treatments may be altered (Booker et al 2009). Moreover, because symptoms can develop slowly, patients may not recognise their impact unless questioned. While there is much evidence on the role of exercise to reduce cancer related fatigue, little is known of the role of exercise in myeloma. However, a recent study with 37 myeloma patients who underwent stretching, aerobic and resistance exercises showed reduced fatigue over time (Groeneveldt et al 2013). Nurses need to provide clear advice to patients on what types of exercise are suitable and to avoid any strenuous activities such as heavy lifting and bending (Crotty 2004).

A reduction in white cells can lead to recurring infections. Recurrent infections are common among myeloma patients and many may die as a result of bacterial and fungal infections. Patient education is therefore essential, alongside prompt recognition of infection and immediate intervention. This education can be provided on an ongoing basis by the ward nurse; it is not enough to just raise it occasionally; patient education for these patients should be embedded in daily practice.

Many myeloma patients are on routine prophylaxis therapy such as antiviral and antifungal agents to prevent infections. In addition, patients receive prophylactic antibiotics where the treatment regime is considered high risk e.g. ASCT. 
Furthermore, patients with hypogammaglobulinemia (low blood immunoglobulin levels) and recurrent infections may benefit from monthly intravenous immunoglobulin (Nucci and Anaissie 2009).

Vaccinations for influenza and pneumonia are also recommended, but may mount a poor response. Notably, varicella-zoster virus reactivation occurs in $10 \%-60 \%$ of patients treated with bortezomib. To prevent these reactivations, antiviral prophylaxis (e.g. acyclovir 500 mg twice daily) has been found effective (Vickrey et al 2009).

Treatment regimens that include bortezomib may reduce platelet levels, resulting in an increased tendency to bruise or bleed. A transfusion of platelets may therefore be required, especially in the early stages of treatment when the treatment is beginning to take effect. However, a complicating factor with myeloma is the increased risk of venous thromboembolism (VTE) with the use of thalidomide and lenalidomide, requiring anti-thrombosis therapy (Alexander et al 2012).

\section{Time out 4}

Think about any tips you would give to patients who are worried about their blood counts.

There are some practical pieces of advice that specialist and clinic nurses can give patients about living with less than optimum blood counts. These include engaging in gentle physical exercise and being careful when planning activities to avoid 
anything too strenuous that may result in over-fatigue. Avoidance of any activities that might increase risk of bruising or bleeding is also important. Nurses should also regularly remind patients to be vigilant to any symptoms of excessive tiredness, infection or feeling unwell and report them immediately.

\section{Time out 5}

Treatment-related peripheral neuropathy is a main concern for myeloma patients. Reflect on your knowledge of the signs and symptoms of PN and what information should be given to patients about treatment related PN.

\section{Peripheral neuropathy}

Peripheral neuropathy (PN) can occur because of myeloma or its treatment. Treatment related peripheral neuropathy is the main cause of PN among myeloma patients (Mothy et al 2010); thalidomide-induced peripheral neuropathy (TiPN) and bortezomib-induced peripheral neuropathy(BiPN) being the leading cause. There is less peripheral neurotoxcity among patients treated with the thalidomide analogues, lenalidomide and pomalidomide (Cundari and Cavaletti 2009). However, lenalidomide can have an effect on stem cell mobilisation whereas bortezomib does not have this effect (Rajkumar 2013).

Bortezomib-induced PN can be sharp, tingling or burning, is more sensory than motor, and can develop suddenly (Cata et al 2007, Delforge et al 2010). Subcutaneous bortezomib is now recommended to minimise PN (Rajkumar 2012), and there is a lower incidence and severity of PN if bortezomib is administered 
subcutaneously (Moreau et al, 2011b). In addition, patient response to subcutaneous administration of bortezomib is comparable to intravenous bortezomib (Moreau et al 2011b). It has also been shown that severe skin reaction can be avoided if the injection site is rotated around eight different sites in the abdomen and thigh, with equivalent serum concentrations on thigh and abdomen (Kamimura et al 2013). However, recent evidence suggests that the site of choice is the abdomen because a higher rate of injection site reaction occurs if given into the thigh, where there is less adipose tissue (Kamimura et al 2013). Should a reaction occur at the injection site, early treatment with topical steroids is advised (Kamimura et al 2012).

Nurses should also advise patients to eat a well-balanced diet comprising of fruit, vegetables, protein and carbohydrates (Miceli et al 2011). And, although the evidence is limited, the International Myeloma Foundation Nurse Leadership Board recommends that myeloma patients should not take Vitamin C, alpha lipoic acid or green tea on the days they receive bortezomib therapy to avoid interference of bortezomib's activity (Miceli et al 2011) (these have been recommended to treat peripheral neuropathy).

Early identification of PN is important because it is reversible if guidelines on dose reduction are followed appropriately. Specialist nurses play an important role in assessing for the presence of PN among all myeloma patients, especially those with pre-existing PN in view of their higher risk of neurological damage (Delforge et al 2010). However, there is no agreement on the best method of assessing PN and 
neuropathic pain (Mateos 2012). This is not surprising because of the subjective nature of patients' pain and numbness. Nevertheless, an 11-item neurotxicity assessment tool originally developed by Calhoun et al (2003) is a useful clinical assessment tool for nurses because of its ease of completion. (Figure 1). 
Figure 1: Neurotoxicity scale (Calhoun et al 2003)

By circling one number per line, please indicate how true each statement has been for you during the past seven days using the following scale:

$0=$ Not at all

$1=$ A little bit

$2=$ Somewhat

3 = Quite $a$ bit

$4=$ Very much

\begin{tabular}{|llllll|}
\hline I have numbness or tingling in my hands & $\mathbf{0}$ & $\mathbf{1}$ & $\mathbf{2}$ & $\mathbf{3}$ & $\mathbf{4}$ \\
I have numbness or tingling in my feet & $\mathbf{0}$ & $\mathbf{1}$ & $\mathbf{2}$ & $\mathbf{3}$ & $\mathbf{4}$ \\
I feel discomfort in my hands & $\mathbf{0}$ & $\mathbf{1}$ & $\mathbf{2}$ & $\mathbf{3}$ & $\mathbf{4}$ \\
I feel discomfort in my feet & $\mathbf{0}$ & $\mathbf{1}$ & $\mathbf{2}$ & $\mathbf{3}$ & $\mathbf{4}$ \\
I have joint pain or muscle cramps & $\mathbf{0}$ & $\mathbf{1}$ & $\mathbf{2}$ & $\mathbf{3}$ & $\mathbf{4}$ \\
I feel weak all over & $\mathbf{0}$ & $\mathbf{1}$ & $\mathbf{2}$ & $\mathbf{3}$ & $\mathbf{4}$ \\
I have trouble hearing & $\mathbf{0}$ & $\mathbf{1}$ & $\mathbf{2}$ & $\mathbf{3}$ & $\mathbf{4}$ \\
I get a ringing or buzzing in my ears & $\mathbf{0}$ & $\mathbf{1}$ & $\mathbf{2}$ & $\mathbf{3}$ & $\mathbf{4}$ \\
I have trouble buttoning buttons & $\mathbf{0}$ & $\mathbf{1}$ & $\mathbf{2}$ & $\mathbf{3}$ & $\mathbf{4}$ \\
I have trouble feeling the shape of small objects when they & $\mathbf{0}$ & $\mathbf{1}$ & $\mathbf{2}$ & $\mathbf{3}$ & $\mathbf{4}$ \\
are in my hand & & & & & \\
I have trouble walking & $\mathbf{0}$ & $\mathbf{1}$ & $\mathbf{2}$ & $\mathbf{3}$ & $\mathbf{4}$ \\
\hline
\end{tabular}

\section{Pain management}

Pain is one of the commonest symptoms experienced by myeloma patients and often it may have been the reason for initial presentation, or a sign of subsequent 
relapse. Up to $67 \%$ of patients report pain at diagnosis, although this may have been present for several months before (Kariyawasan et al 2007).

At diagnosis, pain may be due to the disease process itself (predominantly from destructive bone disease, but occasionally from plasmacytomas [malignant plasma cell tumours] directly affecting neural tissues). Later in the course of the disease, pain often arises as a side-effect of therapies, e.g. thalidomide or bortezomib neuropathy.

Pain related to bone disease causes significant morbidity and impacts on quality of life for myeloma patients. Pain affects patients' level of activity which in turn has a negative effect on performance status, mood and sleep (Coleman et al 2011).

It is important that nurses ask patients about pain, as referral to occupational therapy or physiotherapy may be helpful alongside disease-directed therapy. Assessment of pain as well as pharmacological and nonpharmacological interventions for pain relief should be employed. Guidelines for pain management of multiple myeloma have recently been published (Snowden et al 2011). Additionally, radiotherapy is effective for treating pain caused by bone lesions. Finally, all nurses caring for myeloma patients need to remain cognisant that pain accompanied by weakness and numbness to limbs may be present due to metastatic spinal cord compression. This is an oncologic emergency and requires rapid initiation of appropriate treatment to significantly improve patient outcome. Therefore, nurses should inform myeloma patients of the importance of reporting any back pain immediately. 


\section{Bone health}

Most myeloma patients will develop osteolytic bone lesions because of the increased activity of bone osteoclasts without an equivalent activity in the bone generating cells, the osteoblasts. The most frequent sites of these lesions are the vertebrae (65\%), ribs (45\%), skull (40\%), shoulders (40\%), pelvis (30\%) and long bones $(25 \%)$ (Zamagni and Cavo 2012). Skeletal involvement results in significant morbidity and pain for myeloma patients. Day to day independence is compromised and increased need for hospitalisation increases treatment costs.

Management of myeloma related bone disease is centred on management of the disease itself, and the use of the targeted therapies mentioned earlier all help reduce the risk of further bone disease and fractures (Miceli et al 2011). The goals of treatment include symptom relief, minimising complications and preservation of patient functioning.

Radiation and surgical procedures can also be used to manage pain and reduce risk of fracture (Miceli et al 2011). Ongoing developments in bone health for myeloma patients include investigations on the use of denosumab, a monoclonal antibody that is effective for pain and prevention of skeletal events (Peddi et al 2013).

Bisphosphonate treatment plays a central role in managing myeloma (Kleber et al 2012). Bisphosphonate drugs used include sodium Clodronate, Disodium Pamidronate, Zoledronic acid and Ibandronic acid (Morris and Cruickshank 2010). 
These drugs are effective inhibitors of bone loss. Recently bisphosphonate therapies have been identified as having an anti myeloma effect when combined with other therapies and are considered to improve survival and reduce tumour burden (Morgan et al 2012).

Bisphosphonate therapy is recommended for all patients with symptomatic myeloma, whether or not bone lesions are evident (Bird et al 2011). The International Myeloma Working Group recommends that patients without active bone disease should discontinue bisphosphonates after two years (International Myeloma Foundation 2010).

However, reports began surfacing in 2003 about the occurrence of osteonecrosis of the jaw (ONJ) among patients treated with intravenous bisphosphonates, with the reported incidences ranging between 2\% and 13\% (Morris and Cruickshank 2010). Clinic nurses who administer IV bisphosphonates need to be aware of OJN, clinical signs for OJN and its prevention and management (Morris and Cruickshank 2010) (Table 2).

\section{Time out 6}

Early recognition of OJN is essential. Therefore nurses need to know its clinical signs. Pause for a moment and write down the signs and symptoms of OJN in myeloma patients. 
Table 2 Signs and Symptoms of osteonecrosis of the jaw (adapted from Dickinson et al 2009)

\begin{tabular}{|l|l|}
\hline \multicolumn{1}{|c|}{ Symptoms } & \multicolumn{1}{c|}{ Signs } \\
\hline Tooth, jaw or facial pain & Painful exposed bone \\
\hline Difficulty with eating and/or speaking & Necrotic bone on view \\
\hline Difficulty performing oral care & Cutaneous or mucosal fistulas \\
\hline Asymptomatic & Loose teeth \\
\hline & $\begin{array}{l}\text { Degeneration of bone and/or } \\
\text { separation of dead bone from healthy } \\
\text { bone seen on } x \text {-ray }\end{array}$ \\
\hline
\end{tabular}

Recommendations for the management of OJN include a dental evaluation and any required dental work before commencing long-term bisphosphonate treatment and effective oral care (Morris and Cruickshank 2010). The patient's dentist should be informed of bisphosphonate treatment and provided with ONJ guidelines with the first referral. Nurses should also remind patients to ensure they inform their dentist of their treatment and to update their haematologist if dental work is required. Nurses also should reinforce self-care measures such as good oral hygiene when taking bisphosphonates (Miceli et al 2011).

Patients should have dental check-ups every six months during treatment (Dickinson et al 2009). The risk of OJN increases when patients receive monthly IV treatment beyond twelve months, so decisions to continue or modify treatment should be made annually (Dickinson et al 2009).

Early recognition of OJN and other skeletal events is essential. Clinic nurses play a key role educating patients on the role of bisphosphonate therapy in their treatment 
plan and providing ongoing support (Maxwell 2007). Assessment at baseline and throughout treatment is necessary to ensure optimal care for patients (Maxwell 2007). Asking patients to keep a diary and record pain scores, pain relief measures, changes in mobility, any new skeletal events, serum creatinine levels and adverse events is recommended (Maxwell, 2007).

\section{Renal complications}

All myeloma patients either have renal complications or are at risk of developing renal complications (Faiman et al 2011). In addition, certain treatments and conditions may also contribute to myeloma patients' risk of renal complications (Box $3)$.

\section{Time out 7}

From your own experience of caring for myeloma patients, what drugs or conditions do you know of that can contribute to myeloma patients' risk of renal complications?

\section{Box 3. Drugs or conditions that may contribute to renal complications in myeloma (Faiman et al 2011)}

- Aminoglycoside antibiotics

- Co-morbidities (e.g. Diabetes, hypertension, increased age)

- Dehydration

- Hypercalcaemia

- Non-steroidal anti-inflammatory agents including cyclooxygen-ase-2 inhibitors

- Progressive disease or cast neuropathy (particularly light chains)

- Radio-contrast dyes or IV contrast agents (e.g. for computed tomography scans) 
Treating myeloma with novel agents can reduce tumour burden and therefore reduce the risk of renal complications. Moreover, evidence suggests that bortezomib-based chemotherapy may restore renal function is some patients with renal failure (Ludwig et al 2007). However, because lenalidomide is excreted via the kidneys, dose reductions are recommended at the beginning of treatment in patients with moderate or severe renal impairment (Dimopoulos et al 2012). In addition, managing hypercalcaemia with adequate hydration, cortiocosteroids and bisphosphonates will also reduce the risk of renal complications (Faiman et al 2011). Dialysis is required if a patient's GFR (glomerular filtration rate) is critically low and patients require close monitoring to ensure early referral for dialysis if needed (Faiman et al 2011).

Specialist nurses play a unique role in early identification of renal complications and ensuring regular monitoring of serum calcium and creatine levels. In addition, all nurses need to advise patients regarding taking adequate fluids and avoiding certain medications such as those mentioned in Box 3 (Faiman et al 2011).

\section{Key nursing role}

Nurses play a key role in promoting quality of life for patients with myeloma. They act as patient advocates in providing education on the disease and the treatment choices available to patients, which allows informed and shared decision-making. When older persons with cancer are included in decision making, they find it supportive and it helps maintain their autonomy (Hughes et al 2009). 
Myeloma is a complicated disease requiring patients to undergo numerous tests and treatments that brings them in contact with a variety of health personnel. Nurses can help patients navigate the hospital system by mapping out their treatment journey and ensuring ongoing assessment of disease and treatment related side effects. Nurses also ensure co-ordination of supportive care strategies such as transfusions.

Effective communication is central to myeloma patients' care. Myeloma patients often feel isolated because of the 'unknown' nature of their illness (Kelly and Dowling 2011) and they need psychosocial support. Often older aged patients can assume a stoic approach to their illness and treatment but it is important that nurses regularly reassure them to talk about how they feel. Listening to patients takes time but the outcome of being heard can change the illness experience and promote resilience. Listening also allows health care professionals to understand better the challenges patients face and is a fundamental skill in a therapeutic relationship. This latter point cannot be stressed enough; older people living with a diagnosis of cancer can experience extreme feelings of disempowerment when they hand over their trust to health care professionals (Hughes et al 2009).

Being able to effectively respond to patients' questions and fears is an important nursing role. Patients' fears and worries are often revealed in their narratives shared through conversations with nurses. These narratives can provide nurses with important information that can be used to improve nursing care (Price 2011). Each patient with myeloma needs to be provided with individually tailored information on 
their disease. To respond effectively, nurses need to keep up-to-date on the treatment options available and their associated toxicities. Equipped with an evidence based knowledge base, nurses can effectively help patients in their decision-making process. Moreover, nurses are ideally placed to educate patients on minimising the risk of life-threatening complications and being vigilant to early signs of them developing.

\section{Conclusion}

Patients with myeloma are typically living longer; therefore nurses need to also pay attention to preventing co-morbid conditions as well as managing treatment related issues (Bilotti et al 2011). Information and support for patients and their carers is essential if patients are to come to terms with their diagnosis and make informed decisions about treatments. Additionally it will enable them to understand the importance of compliance with treatment regimens that can sometimes be demanding. At a minimum, it is important for patients and their families to understand the disease and the aims and risks of treatment. They need to understand that although treatment is not curative, it will relieve symptoms, prolong survival and improve quality of life.

Development of an individualised care plan for each patient is useful. This care plan should consider co-morbidities and risk factors such as age, gender, medications and mobility levels (Miceli et al 2011). An example of such a care plan is provided by Miceli et al (2011). Such care plans not only allow the patient record blood results 
and recent treatments, it also provides information on the reason for the tests and medications prescribed and emphasises patients' monitoring of self-care strategies.

Myeloma can be a lonely disease for patients and they value the support of others (Kelly and Dowling 2011). Patients with myeloma should be aware of support networks in the community; whether these are specific to myeloma or in relation to cancer generally. There are also a number of specific support avenues including the International Myeloma Foundation (IMF), Myeloma UK, MyMyeloma Ireland and the Myeloma Research Foundation (USA). (Box 4).

In conclusion, myeloma is an individual cancer affecting patients and carers in many physical, emotional and social ways. Information and support should therefore, if possible, be tailored to individual needs. Nurses play a central role in delivering and co-ordinating the information and support needed to ensure that the best possible quality of life is achieved for each patient living with a diagnosis of myeloma. 
Box 4 Useful contacts

International Myeloma Foundation (IMF): http://www.myeloma.org/

Myeloma UK: http://www.myeloma.org.uk/

Myeloma Ireland: http://www.mymyeloma.ie/

Myeloma Euronet: http://www.myeloma-euronet.org

The Multiple Myeloma Research Foundation: http://www.themmrf.org/ 


\section{References}

Alexander M, Kirsa S, Mellor JD. (2012) Thalidomide thromboprophylaxis in multiple myeloma: A review of current evidence. Asia-Pacific Journal of Clinical Oncology.8(4):319-24.

Bilotti E, Gleason CL, McNeill A. (2011) Routine health maintenance in patients living with multiple myeloma: Survivorship care plan of the international myeloma foundation nurse leadership board. Clinical Journal of Oncology Nursing.15, SUPPL., 25-40.

Bird JM, Owen RG, D'Sa S, Snowden JA, Pratt G, Ashcroft J, et al. (2011) Guidelines for the diagnosis and management of multiple myeloma 2011. British Journal of Haematology.154, 1, 32-75.

Booker R, Olson K, Pilarski LM, Noon JP, Bahlis NJ. (2009) The relationships among physiologic variables, quality of life, and fatigue in patients with multiple myeloma. Oncology Nursing Forum. 36, 2, 209-16.

\section{Calhoun EA, Welshman EE, Chang CH, Lurain JR, Fishman DA, Hunt TL, Cella D} (2003) Psychometric evaluation of the Functional Assessment of Cancer Therapy/Gynecologic Oncology Group - Neurotoxicity (Fact/GOG-Ntx) questionnaire for patients receiving systemic chemotherapy. International Journal of Gynecological Cancer, 13, 741-748.

Cata JP, Weng HR, Burton AW, Villareal H, Giralt S, Dougherty PM. (2007) Quantitative Sensory Findings in Patients With Bortezomib-Induced Pain. Journal of Pain. 8, 4, 296-306.

Chang H (2012) Maintenance therapy for multiple myeloma (ASCO 2012) The Myeloma Beacon. http://www.myelomabeacon.com/news/2012/07/27/maintenancetherapy-for-multiple-myeloma-asco-2012/ accessed 18/7/2013

Coleman EA, Goodwin JA, Coon SK, et al. (2011). Fatigue, sleep, pain, mood, and performance status in patients with multiple myeloma. Cancer Nursing.34, 3, 219-27.

Crotty G (2004) Multiple myeloma- patient information and support and palliative therapy. Irish Medical Times November 19 $19^{\text {th. }} 50$

Cundari S, Cavaletti G. (2009) Thalidomide chemotherapy-induced peripheral neuropathy: Actual status and new perspectives with thalidomide analogues derivatives. Mini-Reviews in Medicinal Chemistry. 9, 7, 760-768. 
Delforge M, Bladé J, Dimopoulos MA, et al. (2010) Treatment-related peripheral neuropathy in multiple myeloma: the challenge continues. The Lancet Oncology. 11,11,1086-1095.

Dickinson M, Prince HM, Kirsa S, et al. (2009) Osteonecrosis of the jaw complicating bisphosphonate treatment for bone disease in multiple myeloma: An overview with recommendations for prevention and treatment. Internal Medicine Journal. 39, 5, 304-16.

\section{Dimopoulos M A, Terpos E, Goldschmidt H, Alegre A, Mark, T, Niesvizky R.} (2012). Treatment with lenalidomide and dexamethasone in patients with multiple myeloma and renal impairment. Cancer Treatment Reviews. 38,8, 1012-1019.

Engelhardt M, Kleber M, Udi J, et al. (2010) Consensus statement from European experts on the diagnosis, management, and treatment of multiple myeloma: From standard therapy to novel approaches. Leukemia and Lymphoma.51, 8, 1424-43.

Faiman BM, Mangan P, Spong J, Tariman JD. (2011) Renal complications in multiple myeloma and related disorders: Survivorship care plan of the international myeloma foundation nurse leadership board. Clinical Journal of Oncology Nursing.15 (SUPPL.), 66-76.

George ED, Sadovsky R. (1999) Multiple myeloma: Recognition and management. American Family Physician. 59, 7, 1885-92.

Groeneveldt L, Mein G, Garrod R, , et al. (2013) A mixed exercise training programme is feasible and safe and may improve quality of life and muscle strength in multiple myeloma survivors. BMC Cancer.13.

Harousseau JL. (2012) How to select among available options for the treatment of multiple myeloma. Annals of Oncology.23, SUPPL. 10, x334-x8.

Hughes N, Closs JS, Clark D. (2009). Experiencing cancer in old age: A qualitative systematic review. Qualitative Health Research. 19, 8, 1139-1153.

International Myeloma Foundation (2010) IMWG guidelines for the use of bisphosphonates in myeloma. http://myeloma.org/ArticlePage.action?tabId $=4 \&$ menuId $=360 \&$ articleId $=2885 \& \mathrm{aTab}=-$ 1 \&gParentType $=$ menuitem \&gParentId $=360 \&$ parentIndexPageId $=47 \&$ parentCategoryId $=72$ $\underline{3}$ accessed $19^{\text {th }}$ July 2013 
Jacobson S, Eggert J, Smith K, Rosenzweig T. (2012) Cytogenetics: Oncology nurses in advanced practice decipher del(13q14) and t(14:16). Clinical Journal of Oncology Nursing.16, 6, E221-E5.

Kamimura T, Miyamoto T, Takashima S, et al. (2012) Injection site reaction after subcutaneous administration of bortezib in Japanese patients with multiple myeloma. International Journal of Hematology.96, 4, 525-7.

Kamimura T, Miyamoto T, Yokota N, et al (2013) Higher incidence of injection site reactions after subcutaneous bortezomib administration on the thigh compared with the abdomen. European Journal of Haematology.90, 2, 157-61.

Kariyawasan, C.C., Hughes, D.A., Jayatillake, M.M. \& Mehta, A.B. (2007) Multiple myeloma: causes and consequences of delay in diagnosis. Quarterly Journal of Medicine, 100, 635-664.

Kelly M, Dowling M (2011) Living with a diagnosis of myeloma: an 'unknown' cancer. Nursing Standard. 25, 8, 38-45.

Kleber M, Udi J, Metzke B, et al. (2012) Challenging the current approaches to multiple myeloma- and other cancer-related bone diseases: From bisphosphonates to targeted therapy. Leukemia and Lymphoma. 53, 6, 1057-61.

Kumar S, Dispenzieri A, Lacy MQ, et al (2007) Impact of lenalidomide therapy on stem cell mobilization and engraftment post-peripheral blood stem cell transplantation in patients with newly diagnosed myeloma. Leukemia, 21, 2035-2042.

Ludwig H, Drach J, Graf H, Lang A, Meran JG. (2007) Reversal of acute renal failure by bortezomib-based chemotherapy in patients with multiple myeloma. Haematologica. 92, 10, 1411-4.

Mateos MV. (2012) How to maintain patients on long-term therapy: Understanding the profile and kinetics of adverse events. Leukemia Research.36, SUPPL.1, S35-S43.

Maxwell C. (2007) Role of the nurse in preserving patients' independence. European Journal of Oncology Nursing. 11, SUPPL. 2, S38-S41.

Miceli TS, Colson K, Faiman BM, Miller K, Tariman JD. (2011) Maintaining bone health in patients with multiple myeloma: Survivorship care plan of the international myeloma foundation nurse leadership board. Clinical Journal of Oncology Nursing.15, Suppl, 9-23. 
Mohty B, El-Cheikh J, Yakoub-Agha I, Moreau P, Harousseau JL, Mohty M. (2010) Peripheral neuropathy and new treatments for multiple myeloma: Background and practical recommendations. Haematologica. 95, 2, 311-319.

Moreau P, Attal M, Pégourié B, et al. (2011a) Achievement of VGPR to induction therapy is an important prognostic factor for longer PFS in the IFM 2005-01 trial. Blood. 117, 11, 3041-3044.

Moreau P, Pylypenko H, Grosicki S, et al. (2011b) Subcutaneous versus intravenous administration of bortezomib in patients with relapsed multiple myeloma: $A$ randomised, phase 3, non-inferiority study. The Lancet Oncology.12, 5, 431-40.

Morgan GJ, Davies FE, Gregory WM, et al. (2012) Effects of induction and maintenance plus long-term bisphosphonates on bone disease in patients with multiple myeloma: The Medical Research Council Myeloma IX Trial. Blood.119, 23, 5374-83.

Morris M, Cruickshank S. (2010) Bisphosphonate-related osteonecrosis of the jaw in cancer patients: Implications for nurses. European Journal of Oncology Nursing.14, 3, 205-10.

Muta T, Miyamoto T, Fujisaki T, et al. (2013) Evaluation of the feasibility and efficacy of autologous stem cell transplantation in elderly patients with multiple myeloma. Internal Medicine.52, 1, 63-70.

National Institute for Health and Clinical Excellence (NICE) (2008) Epoetin alfa, epoetin beta, and darbepoetin alfa for cancer treatment-induced anaemia. Final Appraisal Determination. London, UK: NICE.

Nucci M, Anaissie E. (2009) Infections in patients with multiple myeloma in the era of high-dose therapy and novel agents. Clinical Infectious Diseases. 49, 8, 1211-25.

Oken MM, Creech RH, Torme, DC, et al. (1982) Toxicity And Response Criteria Of The Eastern Cooperative Oncology Group. Am J Clin Oncol 5, 649-655.

Palumbo, A. (2012). Novel agents and the paradigm of continuous treatment in multiple myeloma. Leukemia Research. 36, Suppl 1, S1-S2. 
Palumbo A, Bringhen S, Ludwig $\mathbf{H}$, et al. (2011) Personalized therapy in multiple myeloma according to patient age and vulnerability: A report of the European Myeloma Network (EMN). Blood.118, 17, 4519-29.

Peddi P, Lopez-Olivo MA, Pratt GF, Suarez-Almazor ME. (2013) Denosumab in patients with cancer and skeletal metastases: A systematic review and meta-analysis. Cancer Treatment Reviews.39, 1, 97-104.

Price B. (2011) Making better use of older people's narratives. Nursing older people. 23, 6, 31-7.

Quach H, Kalff A, Spencer A. (2012) Lenalidomide in multiple myeloma: Current status and future potential. American Journal of Hematology.87,12, 1089-95.

Rajkumar SV (2012) Multiple myeloma: 2012 update on diagnosis, risk-stratification, and management. American Journal of Hematology. 87, 78-88.

Rajkumar VS. (2013) Multiple myeloma: 2013 update on diagnosis, riskstratification, and management. American Journal of Hematology.88, 3, 225-35.

Seiter K (2013) Multiple myeloma. Treatment and Management. Medscape. http://emedicine.medscape.com/article/204369-overview accessed 2/5/2013

Shimoni A, Hardan I, Ayuk F et al. (2010) Allogenic hematopoietic stem-cell transplantation with reduced-intensity conditioning in patients with refractory and recurrent multiple myeloma: Long-term follow-up. Cancer.116, 15, 3621-30.

Snowden JA, Ahmedzai SH, Ashcroft J. et al (2011) Guidelines for supportive care in multiple myeloma 2011. British Journal of Haematology : 154, 1, 76-103.

Vickrey E, Allen S, Mehta J, Singhal S. (2009) Acyclovir to prevent reactivation of varicella zoster virus (herpes zoster) in multiple myeloma patients receiving bortezomib therapy. Cancer. 115, 1, 229-32.

Zamagni E, Cavo M. (2012). The role of imaging techniques in the management of multiple myeloma. British Journal of Haematology. 159, 5, 499-513. 